\author{
JOANNA TARGOŃSKA \\ Uniwersytet Warmińsko-Mazurski w Olsztynie \\ joanna.targonska@uwm.edu.pl \\ ORCID: 0000-0001-5495-3358
}

\title{
Kollokationskompetenz vs. Sprachfertigkeiten bzw. andere Sprachkompetenzen - ein Forschungsüberblick
}

\author{
Collocational competence and other types \\ of skills and competences: \\ An overview of the state of the art
}

\begin{abstract}
The goal of the article is to examine the dependency between collocational competence and other competences and language skills of a foreign language learner. The paper begins with an explication of the notions of collocation and collocational competence. It further presents the results of an analysis of the relevant research on collocational competence. Based on publications of researchers from all over the world, the research question which has been formulated concerns the dependency between a well developed collocational competence and the level of proficiency in reading, listening, writing and speaking. And conversely, the analysis seeks to find out if the language skills above automatically develop learners' collocational competence. Finally, the article shows the influence of collocational competence on language reception and production, and discusses some methods which may improve foreign language learners' collocational competence.
\end{abstract}

KEYWORDS: collocation, collocational competence, vocabulary learning, collocational awareness, vocabulary competence, vocabulary work.

SCHLÜSSELWORTE: Kollokation, Kollokationskompetenz, Wortschatzlernen, Kollokationsbewusstsein, Wortschatzkompetenz, Wortschatzarbeit. 


\section{EINLEITUNG}

Kollokationen rücken als Forschungsgegenstand immer mehr ins Zentrum des Interesses. Dabei handelt es sich nicht nur um rein linguistische (immer häufiger korpuslinguistische) Studien, sondern um eine am Fremdsprachenerwerb orientierte Forschung, d.h. um Überlegungen zum Erwerb bzw. Lernen von Kollokationen und deren Speicherung im mentalen Lexikon der Fremdsprachenlernenden. Gerade in den letzten zehn Jahren ist die Anzahl der empirischen Studien zum Stand und Erwerb der Kollokationskompetenz der Fremdsprachenlernenden, besonders zum Einfluss verschiedener didaktischer Schritte und Methoden auf die Qualität des Erwerbs des neuen Vokabulars sowie die Quantität der beherrschten Kollokationen, rapide gestiegen. Jede Studie stellt nur ein kleines Feld der Kollokationsforschung dar. Anhand der Ergebnisse vieler kleiner empirischer Studien kann man sich nicht immer ein vollständiges Bild von dem Erwerb bzw. von der Entwicklung der Kollokationskompetenz machen.

Das Ziel des Beitrags, der sich mit der Kollokationskompetenz beschäftigt, ist es zu untersuchen, welche Rolle die Kollokationskompetenz beim Fremdsprachenlernen, d.h. bei der Entwicklung aller Sprachfertigkeiten spielt und ob sich die Kollokationskompetenz und andere Sprachfertigkeiten bzw. -kompetenzen ${ }^{1}$ gegenseitig beeinflussen. Da in den letzten zehn Jahren zahlreiche empirische Studien an Fremdsprachenlernenden von Wissenschaftlern aus der ganzen Welt durchgeführt wurden, werden im vorliegenden Artikel keine neuen Daten erhoben, sondern die bereits vorhandenen Untersuchungen auf den Zusammenhang zwischen der Kollokationskompetenz und anderen Sprachfertigkeiten analysiert. Im Zentrum des Interesses steht die gegenseitige Beeinflussung der Kollokationskompetenz, als Teil der Wortschatzkompetenz (vgl. Targońska \& Stork 2013), und der anderen rezeptiven sowie produktiven Sprachfertigkeiten. Dabei soll anhand empirischer Studien anderer Autoren zum einen geprüft werden, ob und inwieweit eine gut entwickelte Kollokationskompetenz weitere Sprachkompetenzen bzw. Sprachfertigkeiten positiv beeinflussen kann. Zum anderen steht im Zentrum des Interesses die Frage, ob die Ergebnisse der schon durchgeführten Studien darauf hinweisen, dass die Arbeit an bestimmten Sprachfertigkeiten automatisch zum Erwerb der Kollokationskompetenz führt bzw. führen kann. Im Anschluss an die dargestellten Ergebnisse der Analyse werden

\footnotetext{
${ }^{1}$ Im Folgenden wird ein weites Kompetenzkonzept vertreten (vgl. Hug \& Leupold 2008: 55ff.), in dem auch alle Sprachfertigkeiten als Kompetenzen aufgefasst werden. Man kann von produktiven und rezeptiven Sprachfertigkeiten bzw. Kompetenzen sprechen, d.h. z.B. sowohl von der Fertigkeit Leseverstehen als auch von der Kompetenz Leseverstehen.
} 
die in diesem Beitrag aufgestellten Hypothesen zur möglichen Interaktion zwischen der Kollokationskompetenz und anderen Sprachfertigkeiten präsentiert.

Der Beitrag ist wie folgt gegliedert: Ausgegangen wird von den Termini Kollokation und Kollokationskompetenz. Dabei wird auf unterschiedliche Auffassungen des Kollokationsbegriffs und die daraus resultierenden Probleme hingewiesen. Dargestellt wird die Kollokationskompetenz in ihrer Dreigliedrigkeit. Weiterhin wird die gegenseitige Beeinflussung der Kollokationskompetenz und der rezeptiven Sprachfertigkeiten (Lese- und Hörverstehen) unter die Lupe genommen, wobei Ergebnisse der schon durchgeführten empirischen Studien den Untersuchungsgegenstand darstellen. Dem folgt die Analyse der Interaktion zwischen der Kollokationskompetenz und den produktiven Sprachfertigkeiten (Schreiben und Sprechen). Abgerundet wird der Beitrag mit zusammenfassenden Bemerkungen, wobei die jeweils stärkste und schwächste Korrelation zwischen der Kollokationskompetenz und anderen Sprachkompetenzen präsentiert wird.

\section{ZUM TERMINUS "KOLLOKATION“}

Kollokation ist ein Terminus, der unterschiedlich aufgefasst wird. Aus Platzgründen kann an dieser Stelle auf dessen Entfaltung und Bedeutungsverschiebungen bzw. Anfänge der Kollokationsforschung nicht eingegangen werden (mehr dazu z.B. bei Konecny 2010; Targońska 2014). Eines ist aber festzuhalten: Seit der bahnbrechenden Arbeit von Hausmann (1984) existieren im linguistischen und fremdsprachenerwerbstheoretischen Diskurs zwei Hauptströmungen in der Auffassung des Kollokationsbegriffs. Zum einen kann man das frequenzorientierte, zum anderen das bedeutungsorientierte Verständnis der Kollokationen beobachten. Das erste hat zwar seine Wurzeln im Britischen Kontextualismus und in der Computerlinguistik, wird jedoch ebenso in der Korpuslinguistik eingesetzt. Das zweite, semantikorientierte Verständnis verdanken wir den Überlegungen von Hausmann (1984), der als erster auf die hierarchische Struktur der Kollokationen und die semantische Beeinflussung bzw. gegenseitige semantische Abhängigkeit der Kollokationsglieder hingewiesen hat. In seiner Auffassung haben die Glieder der kollokationalen Wortverbindung konkrete Rollen: die Kollokationsbasis fungiert als das bedeutungstragende und der Kollokator als bedeutungsspezifizierendes Element, das der Kollokationsbasis aufgrund der Konvention der jeweiligen Sprache zugeordnet wird und dieser auch semantisch untergeordnet ist. 
Interessanterweise wird im englischsprachigen linguistischen Diskurs und in Bezug auf Englisch als Fremdsprache die weite, d.h. korpuslinguistische, frequenzorientierte Auffassung des Kollokationsbegriffs angenommen, während in Bezug auf Deutsch als Fremdsprache und andere Fremdsprachen (außer dem Englischen als Fremdsprache) viel häufiger die bedeutungsorientierte Auffassung des Kollokationsbegriffs angenommen wird ${ }^{2}$. Seit der Erweiterung des Phraseologismus-Begriffs um die nicht idiomatischen, festen Wortverbindungen (Phraseologismen im weiteren Sinne) wird die von Hausmann propagierte enge, d.h. semantik- bzw. bedeutungsorientierte Auffassung, auch als phraseologische Auffassung des Kollokationsbegriffs bezeichnet (vgl. Reder 2006; Hausmann 2007)3.

Das zweifache Verständnis des Kollokationsbegriffs bringt viele Probleme mit sich. Dabei handelt es sich erstens um die Nomenklatur. Nicht alle Wortverbindungen, die in der frequenzorientierten Auffassung als Kollokationen gedeutet werden, werden in der phraseologischen, bedeutungsorientierten Auffassung den Kollokationen zugerechnet. Viele von ihnen (z.B. ein Buch lesen) müssen nämlich als freie Wortverbindungen von hoher Frequenz bezeichnet werden, denn jedes Glied dieser Wortverbindung behält in diesem Syntagma seine primäre Bedeutung. Das zweite Problem bezieht sich auf empirische Forschungen und deren Ergebnisse. Zum einen geht aus manchen Untersuchungen nicht hervor, welches Verständnis der Kollokationen einer konkreten Studie zu Grunde liegt. Zum anderen sind die Ergebnisse vieler Studien nicht miteinander vergleichbar, insbesondere dann, wenn sie auf unterschiedlichen Auffassungen des Kollokationsbegriffs basieren. Deshalb sollten Autoren aller empirischen, insbesondere der fremdsprachenerwerbsorientierten Studien zu Kollokationen ihre Auffassung des Kollokationsbegriffs darlegen, was jedoch viele von ihnen nicht tun. Eins steht jedoch fest: Wenn der Erwerb von Kollokationen (in diesen unterschiedlichen Auffassungen) den Untersuchungsgegenstand darstellt, dann handelt es sich zweifelsfrei um den Erwerb von Syntagmen, d.h. von mehr oder weniger festen Wortverbindungen. Problematisch ist jedoch die Tatsache, dass unterschiedliche Auffassungen des Kollokationsbegriffs eine andere Widerspiegelung in kognitiven Prozessen, bzw. im mentalen Lexikon haben. Kollokationen sind in der phraseologischen Auffassung nicht frei kreierbar, sondern nur reproduzierbar, weswegen sie als eine Ganzheit,

\footnotetext{
2 Natürlich finden sich Anhänger der korpuslinguistischen Auffassung des Kollokationsbegriffs in der Gruppe aller Neuphilologien. Als Beispiel kann man hier Siepmann (2002; 2004) nennen, der für einen weiten Kollokationsbegriff plädiert.

${ }^{3}$ Reder (2006: 160) betrachtet Kollokation als „eine phraseologische Einheit." Hausmann (2007: 2018) bezeichnet Kollokationen als „normtypische, phraseologische Wortverbindungen, die aus einer Basis und einem Kollokator bestehen."
} 
d.h. als eine semantische Einheit im mentalen Lexikon gespeichert und in dieser Form aus ihm abgerufen werden sollten ${ }^{4}$. Nur die Speicherung dieser Kollokationen als einer Ganzheit kann zu ihrem korrekten Gebrauch führen. Demgegenüber werden in der frequenzorientierten Auffassung unter dem Kollokationsbegriff oft auch frequente freie Wortverbindungen subsummiert, die frei kreierbar sind. Diese müssen nicht als eine Ganzheit gespeichert werden, denn der Fremdsprachenlernende kann diese Syntagmen aus den Wörtern, die im eigentlichen Sinne gebraucht werden, frei kreieren, indem er die ihm bekannten Einzelwörter zusammenstellt. Diese Kollokationen, die oft freie Wortverbindungen von hoher Frequenz darstellen, können häufig problemlos Wort für Wort aus der Ausgangs- in die Zielsprache übersetzt werden 5 .

\section{ZUM BEGRIFF KOLLOKATIONSKOMPETENZ}

Während also bereits der Terminus Kollokation unterschiedlich aufgefasst wird, ist die Definition des Begriffs Kollokationskompetenz noch schwieriger. Zwar wird dieser schon seit Langem in vielen Forschungsarbeiten gebraucht, jedoch wird er dort nicht explizit erklärt. Er wird bisher in keinem Handbuch zur Fremdsprachendidaktik erfasst (vgl. dazu die Analysen von Targońska 2014) ${ }^{6}$. Die erste mir bekannte quasi explizite Beschäftigung mit der Kollokationskompetenz ist bei Hill (1999) zu ermitteln. Obwohl der Titel seines Artikels "Collocational competence“ lautet, beschäftigt sich der Autor darin in erster Linie mit dem Terminus Kollokation (dabei unterscheidet er unique collocations, strong collocations, weak collocations, medium-

\footnotetext{
${ }^{4}$ Dies ist damit zu erklären, dass die Zuordnung der Kollokationsglieder in den Kollokationen (in der phraseologischen Auffassung) auf der Konvention der jeweiligen Sprache beruht, weswegen eine Kollokation in unterschiedlichen Sprachen mittels unterschiedlicher lexikalischer Elemente realisiert werden kann (oft kann ein Glied der Kollokation nicht durch ein quasi synonymes Wort ersetzt werden; Kollokationsglieder sind also häufig nicht substituierbar), was zu ihrer Unvorhersagbarkeit führt und keine freie Kreierbarkeit (Reproduzierbarkeit) ermöglicht.

${ }^{5}$ Der Autorin des vorliegenden Beitrags ist bewusst, dass manchen freien Wortverbindungen in einer (Fremd-) Sprache (z.B. im Polnischen zrobić zdjęcie, im Deutschen ein Foto machen) in einer anderen Sprache eine Kollokation entspricht (z.B. im Englischen take a photo).

${ }^{6}$ Festzuhalten ist jedoch, dass im Handbuch zum DaF/DaZ (Krumm, Fandrych, Hufeisen \& Riemer 2010) in dem von Tschirner (2010) verfassten Beitrag zum Wortschatz bei der Erläuterung des Begriffs „lexikalische Kompetenz" Kollokationen erwähnt werden. Darin wird darauf verwiesen, dass auf der produktiven Seite des Wortschatz-Verwendungswissens das Wissen dazu gehört, „,welche Wörter mit welchen anderen Wörtern verwendet werden müssen, wenn man idiomatisch richtig sprechen möchte (Kollokationen)“ (Tschirner 2010: 242).
} 
strength collocations). Selbst der Abschnitt zur Kollokationskompetenz liefert keine explizite Definition bzw. Auslegung dieses Begriffs. Hill verweist darin auf Kollokationsprobleme der Fremdsprachenlernenden und auf die große Rolle der Kollokationen, deren Kenntnis den präzisen sprachlichen Ausdruck erlaubt und die Intonation positiv beeinflusst. Den Terminus Kollokationskompetenz haben in der deutschsprachigen Forschung u.a. Bahns (1997), Lütge (2000), Reder (2001; 2002; 2006), Rössler (2010) und Müller (2011) gebraucht. Jedoch ist in ihren Arbeiten keine explizite Definition der Kollokationskompetenz zu finden. Darin erscheinen jedoch solche Begriffe wie: rezeptive und produktive Kollokationskompetenz. Müller (2011) spricht noch vom Kollokationsbewusstsein und bei Viehweger (1987) finden wir den Hinweis auf das Kollokationswissen, das bei der Äußerung aktiviert wird.

Obwohl, wie oben angedeutet, der Begriff Kollokationskompetenz schon seit einigen Jahrzehnten in wissenschaftlichen Abhandlungen immer wieder gebraucht wird, lässt sich die explizite Erläuterung dieses Begriffs erst nach 2010 feststellen. Henriksen (2013: 39f.) definiert die Kollokationskompetenz in Form von Teilfertigkeiten, die die Kollokationskompetenz ausmachen. Dazu gehört nach ihr neben der Fähigkeit, Kollokationen in Texten wahrzunehmen und zu erkennen, auch die der Bedeutungserschließung und die Erkennung der Form-Bedeutung-Funktion-Zusammenhänge. Darüber hinaus äußert sich die Kollokationskompetenz in der Kenntnis der Gebrauchsrestriktionen von Kollokationen. Der Fremdsprachenlernende mit einer gut entwickelten Kollokationskompetenz kann unter verschiedenen Kollokationsoptionen auswählen und in der Sprachkommunikation schnell und flüssig auf diese zurückgreifen. Weiterhin betont Henriksen (2013: 33f.), dass sich die Kollokationskompetenz auf eine breite Palette von Wissen über die kombinatorische Verwendung von lexikalischen Einheiten bezieht. Eine gut entwickelte Kollokationskompetenz ermögliche eine effiziente und flüssige Kommunikation.

In einem neuen Modell der lexikalischen Kompetenz plädieren Targońska und Stork (2013: 93ff.) für die Auffassung der lexikalischen Kompetenz als Bündel von Subkompetenzen, wobei die Kollokationskompetenz einen wichtigen Bestandteil der Wortschatzkompetenz ausmachen soll. Kollokationskompetenz wird in ihrem Modell als keine einheitliche Größe betrachtet, denn innerhalb dieser Kompetenz können drei unterschiedliche Teilkompetenzen unterschieden werden, nämlich die rezeptive, produktive und reflexive (verbunden mit dem Sprachbewusstsein, insbesondere dem Kollokationsbewusstsein) Kollokationskompetenz (Targońska \& Stork 2013: 96f.). Die rezeptive Kollokationskompetenz umfasst die Fähigkeit, die Bedeutung der Kollokation $\mathrm{zu}$ entziffern bzw. diese $\mathrm{zu}$ verstehen sowie nicht korrekt gebildete Kollokationen zu erkennen (Targońska \& Stork 2017: 228). Produktive Kolloka- 
tionskompetenz kommt zum einen in der korrekten Verwendung von Kollokationen zum Ausdruck, zum anderen impliziert sie die Fähigkeit, inkorrekte Kollokationen zu berichtigen. Die reflexive Kollokationskompetenz stellt einen Teil des Sprachbewusstseins (Kollokationsbewusstsein) dar. Sie umfasst die Fähigkeit, unbekannte Kollokationen in Texten zu erkennen und scheint somit die Grundlage für die Entwicklung der rezeptiven und produktiven Kollokationskompetenz darzustellen, weil dank des vorhandenen Kollokationsbewusstseins die Fremdsprachenlernenden eher dazu tendieren sollen, zu einer Kollokation nach einer zielsprachigen Äquivalenz in der Ausgangssprache $\mathrm{zu}$ suchen und nicht automatisch zur Eins-zu-Eins-Übersetzung zu neigen. Weiterhin ist die reflexive Kollokationskompetenz mit dem Sprachlernbewusstsein verbunden, denn dieses schließt "die Bewusstheit der Notwendigkeit der Speicherung von Kollokationen als Lerneinheiten (im Sinne von kollokationalem Lernen)“ ein (Targońska \& Stork 2017: 228).

\section{KOLLOKATIONSKOMPETENZ UND ANDERE SPRACHFERTIGKEITEN}

Wie bereits angekündigt, werden in diesem Teil des Beitrags Ergebnisse der bisher durchgeführten empirisch ausgerichteten Studien zum Lernen von Kollokationen analysiert und dargestellt. Dabei wird der Fokus der Aufmerksamkeit auf die gegenseitige Beeinflussung der Kollokationskompetenz bzw. der Kenntnis von Kollokationen auf bestimmte Sprachfertigkeiten und umgekehrt gerichtet. Im Zentrum des Interesses steht auch die Frage, inwieweit die Arbeit an bestimmten Sprachfertigkeiten zur Entwicklung der Kollokationskompetenz beiträgt bzw. beitragen kann.

\subsection{Kollokationskompetenz vs. Lesen}

Die ersten empirischen Studien zur Kollokationskompetenz, die in den 1970er bis 1990er Jahren durchgeführt wurden, gingen der Frage nach, inwieweit Kollokationen durch Lesen erworben werden (können). Aus diesen Studien geht hervor, dass das Lesen von Texten nicht automatisch zur Beherrschung von Kollokationen führt. Die zuvor unbekannten Kollokationen, deren Bedeutung im Leseprozess korrekt rezipiert wurde, wurden nach dem Lesen eines Textes nicht ad hoc erworben (vgl. Marton 1977; Gabryś-Biskup 1990). Auch die Beherrschung von Kollokationen, mit denen Fremdsprachenlernende im Leseprozess konfrontiert wurden, war sehr gering (vgl. Bahns \& Sibilis 1992). Die Autoren ziehen daraus die Schlussfolgerung, dass zur Beherrschung von Kollokationen beim Lesen eine bewusste Aufmerk- 
samkeitsfokussierung auf Kollokationen notwendig ist (vgl. Bahns 1997: 71). Dies bestätigen auch die Ergebnisse der von Lütge (2002) durchgeführten Untersuchung, aus der hervorgeht, dass Fremdsprachenlernende im Leseprozess den Einzelwörtern mehr Aufmerksamkeit schenken, wobei Syntagmen (Kollokationen stellen eine besondere Art der Syntagmen, der konventionellen bzw. konventionalisierten Syntagmen dar) eher unauffällig zu sein scheinen. Die Steuerung der Aufmerksamkeit der Fremdsprachenlernenden auf Syntagmen kann zwar ihren Erwerb positiv beeinflussen. Jedoch führt die Aufmerksamkeitsfokussierung auf Syntagmen nicht bei allen Fremdsprachenlernenden zu einer besseren Beherrschung, denn es lassen sich dabei große individuelle Unterschiede beobachten. Laut Lütge (2002: 221) ist die Beherrschung von Syntagmen von der Art des Lesens abhängig, wobei formorientiertes Rezipieren von Lesetexten das Wahrnehmen von den in Texten vorkommenden (mehr oder weniger festen) Syntagmen positiv beeinflusst, wodurch der Erwerb von Kollokationen begünstigt werden kann.

Auf die Unauffälligkeit der Kollokationen in Lesetexten weist auch Reder (2006) hin. In ihrer Studie konnte sie beobachten, dass sich die Kollokationskenntnis nach dem Lektürelesen nur um 3\% verbesserte, was sie auf inhaltsorientiertes Lesen der DaF-Lernenden zurückführte, bei dem Kollokationen nicht als eine lexikalische Einheit wahrgenommen, diese also als solche nicht gespeichert werden. Die nach dem Lesen eingesetzten lexikalischen Übungen führten zum Anstieg der internalisierten Kollokationen. Daraus zieht sie die Schlussfolgerung, dass zum Erwerb von Kollokationen explizite Kollokationsübungen notwendig sind, durch die den Fremdsprachenlernenden Kollokationen bewusst gemacht werden.

Die dem Lesen von Texten folgenden Aktivitäten, dank denen neue Kollokationen erworben werden können, können unterschiedlich sein. Zum einen können sie sich auf die Form der Kollokationen (lexikalische Übungen und Aufgaben), zum anderen auf deren Bedeutung (kommunikative Aufgaben) konzentrieren. Aus der Studie von Khonamri und Roostaee (2014: 1041f.) geht hervor, dass die beiden Aktivitäten zum erhöhten Erwerb von den in Lesetexten und den darauf folgenden Aufgaben geübten Kollokationen führen, wobei die Konzentration auf die Form der Kollokationen sich als ein wenig effizienter erwiesen hat.

Darüber hinaus kann die Art und Weise der Präsentation bzw. Hervorhebung von Kollokationen in Lesetexten einen Einfluss auf ihren Erwerb haben. Aus der Studie von Goudarzi (2012: 253f.) ergibt sich, dass Kollokationen am besten gespeichert werden, wenn sie in Form einer Wörterliste mit der L1-L2-Übersetzung an den Text angehängt werden, bzw. wenn diese im Text fett hervorgehoben werden. Die in einem Text ohne irgendwelche Inputverstärkung präsentierten Kollokationen fallen den Fremdsprachenlernenden nicht auf. Sie entziehen sich ihrer Aufmerksamkeit, weswegen ihre 
Speicherung in Form von Chunks nicht zustande kommt. Zur Internalisierung von Kollokationen ist ihre explizite Behandlung notwendig, was auch die Ergebnisse der Studie von Nam (2013) bestätigen. Explizite Instruktionen und Übungen zur Wahrnehmung von Kollokationen führen zur besseren Beherrschung der in Lesetexten vorkommenden Kollokationen (Dentisak 2015: 8).

Die Wechselwirkung zwischen der Kollokationskompetenz und dem Leseverstehen kann auch aus einer anderen Perspektive betrachtet werden. Von Interesse ist auch, ob eine gut entwickelte Kollokationskompetenz zu besseren Ergebnissen im Leseverstehen führt. Die Analyse der empirischen Untersuchungen zu diesem Forschungsschwerpunkt hat ergeben, dass solche Studien immer noch ein Desiderat darstellen. Die einzige ermittelte Studie, die dazu erwähnt werden könnte, ist die von Hsu (2010). Eines der Ziele dieser Studie war die Erforschung der Auswirkungen des expliziten Kollokationsunterrichts auf das Leseverstehen. Aus der Untersuchung geht hervor, dass die explizite Behandlung von Wortschatz, und darunter von Kollokationen, zu besseren Ergebnissen im Leseverstehen sowie zur besseren Speicherung des Vokabulars geführt hat, wobei sich die Behandlung von Kollokationen als effizienter als die Behandlung von Einzelwörtern erwiesen hat. Hsu (2010: 62ff.) zieht aus seiner Studie die Schlussfolgerung, dass sich der Kollokationsunterricht (und damit vielleicht auch eine bessere Kollokationskompetenz) positiv auf das Leseverstehen auswirkt.

Bis heute scheinen keine weiteren expliziten Studien zum Einfluss der Kollokationskompetenz auf Ergebnisse im Leseverstehen durchgeführt worden zu sein. Man kann jedoch die Hypothese aufstellen, dass sich die gut entwickelte Kollokationskompetenz positiv auf Ergebnisse im Leseverstehen auswirken kann. Dieser Annahme liegen folgende Prämissen zugrunde: Das Kollokationsbewusstsein und die Kollokationsbewusstheit können dem Fremdsprachenlernenden beim Erkennen der in Texten auftretenden festen konventionellen Wortverbindungen helfen, die nicht Glied für Glied rezipiert bzw. in die L1 übersetzt werden dürfen. Das Erkennen von Kollokationen, die als eine Einheit wahrgenommen und dekodiert werden sollten, kann bei der Bedeutungserschließung helfen. Aus diesem Grunde wird im Folgenden die Meinung vertreten, dass das korrekte Rezipieren von Kollokationen das Leseverstehen nur positiv beeinflussen kann. Weiterhin kann man im Falle der bekannten Kollokationen (vor allem bei solchen mit einem eingeschränkten semantischen Potential eines Kollokationsgliedes; vgl. Reder 2006: 78f.) beim Erscheinen des ersten Gliedes in Lesetexten schon auf das nächste Kollokationsglied schließen. Auch während des Lesens, kann sich das Kollokationsbewusstsein als sehr hilfreich erweisen, denn der Fremdsprachenlernende kann nach der Semantik einer Kollokation in Wörterbucheinträgen zu beiden Kollokationsgliedern (auf der Makroebene des 
Wörterbuchs) und dann in einem Wörterbucheintrag zu dem bestimmten Kollokationsglied nach der Bedeutung in der festen konventionalisierten Wortverbindung, d.h. in der jeweiligen Kollokation (auf der Mikroebene) suchen. Kollokationen können nicht nur in Texten vorkommen, sondern auch in den Aufgaben, in denen das Leseverstehen geprüft wird. Wenn in diesen Aufgaben Kollokationen auftreten, die den Fremdsprachenlernenden nicht bekannt sind, können diese ein Hindernis bei der Bewältigung der Aufgabe zum Leseverstehen darstellen. Somit kann sich auch hier die Kollokationskenntnis als förderlich und brauchbar erweisen.

\subsection{Kollokationskompetenz vs. Hörverstehen}

Die Wechselwirkung zwischen der Kollokationskompetenz und dem Hörverstehen ist noch weniger erforscht. Der Autorin dieses Beitrags ist nur eine empirische Arbeit bekannt, die diesem Thema gewidmet ist. Hulbert (2015) wollte in ihrer Studie anhand einer Dictogloss-Aufgabe untersuchen, ob Fremdsprachenlernende (hier DaF-Lernende) Kollokationen beim Hören wahrnehmen. Bei den Hörtexten handelte es sich um dreimal langsam vorgelesene Texte, die die Versuchspersonen anhand ihrer Notizen inhaltlich rekonstruieren sollten. Hulbert untersuchte anhand der Analyse dieser Notizen, ob Fremdsprachenlernende Kollokationen in den Hörtexten als eine Ganzheit wahrnehmen, erkennen und diese ausschreiben. Ihre Studie hat ergeben, dass die DaF-Lernenden Kollokationen in Hörtexten sehr selten als eine lexikalische Einheit wahrnehmen, obwohl manche Kollokationen besser als andere wahrgenommen werden, was Hulbert auf die Positionierung des Kollokators im Satz zurückführt. Fremdsprachenlernende schenken im Hörverstehensprozess den Kollokationsbasen (dem bedeutungstragenden Element) mehr Aufmerksamkeit als den Kollokatoren (dem bedeutungsspezifizierenden Element). Die Nähe des Kollokators zur Kollokationsbasis wirkt sich positiv auf die Wahrnehmung der Kollokation als eine Ganzheit in gehörten Texten aus. Hulbert zieht aus ihrer Studie folgende Schlussfolgerung: „Inputbezogenes noticing von Kollokationen findet nur in sehr geringem Umfang statt, wenn die Lernenden ihre Aufmerksamkeit nicht bewusst darauf richten" (Hulbert 2015: 187).

Diese singuläre Studie zeigt, dass durch das Hören die Kollokationskompetenz nicht automatisch entwickelt wird. Dies bedeutet, dass das bloße Hören von den in Hörtexten auftretenden Kollokationen nicht ad hoc zu ihrer Speicherung führt. Damit neue Kollokationen beherrscht werden, ist einerseits das Kollokationsbewusstsein, andererseits eine bewusste Aufmerksamkeitsfokussierung notwendig. 
Wie im Falle des Leseverstehens fehlen auch empirische Studien zum Einfluss des Niveaus der Kollokationskompetenz auf das Hörverstehen. Man kann auch von der Hypothese ausgehen, dass sich Kollokationskenntnisse positiv auf das Hören und Verstehen auswirken können. Diese Annahme basiert auf folgenden Überlegungen der Autorin: Wenn man eine Kollokation kennt, reicht das Erscheinen / Auftreten des ersten Kollokationsgliedes im Hörtext, damit der Hörende das nächste Kollokationsglied voraussagen kann. Der Fremdsprachenlernende kann im Laufe des Hörverstehensprozesses dann für einen Sekundenbruchteil „abschalten“, wodurch seine Speicherkapazität entlastet wird. Im Falle der Kenntnis der im Hörtext vorkommenden Kollokationen muss sich der Fremdsprachenlernende / Hörende nicht zu viel Mühe geben, um eine unbekannte Kollokation zu dekodieren bzw. deren Bedeutung aus dem Kontext zu erschließen, denn die bekannte Bedeutung wird von ihm im Hörverstehensprozess aktiviert. Darüber hinaus kann sich eine gut entwickelte Kollokationskompetenz auch bei der Lösung der Aufgaben zum Hörverstehen als vorteilhaft erweisen, und zwar in unterschiedlichen Aufgabentypen. Bei offenen Fragen, in denen der Fremdsprachenlernende selbstständig eine Antwort geben muss, kann ihm die produktive Kollokationskompetenz beim Formulieren eines korrekten Satzes helfen. Aber auch in Richtig-Falsch-Aufgaben, in denen Kollokationen auftreten, kann ihre Kenntnis bei der Wahl der richtigen Antwort helfen, denn das korrekte Verstehen des zu beurteilenden Satzes hilft bei der Entscheidung über seine (Un-)Korrektheit.

\subsection{Kollokationskompetenz vs. Sprechen}

Aus empirischen Studien zur Wechselwirkung zwischen der Kollokationskompetenz und der Sprechfertigkeit geht hervor, dass das Niveau der Kollokationskompetenz die Sprechfertigkeit positiv beeinflussen kann. Die ersten Studien, in denen die Auswirkung der mangelhaften bzw. mangelnden Kollokationskompetenz nebenbei erfasst wurde, stammen von Zöfgen (2001). Die Untersuchung der mündlichen Äußerung der Fremdsprachenlernenden hat gezeigt, dass es eine große Kluft zwischen der Ausdrucksabsicht der Fremdsprachenlernenden und ihrem Ausdrucksvermögen gibt, wobei fehlende Kollokationskenntnisse hier eine große Rolle spielen. Eine unterentwickelte Kollokationskompetenz der Fremdsprachenlernenden führt zur lexikalischen Reduktion, lexikalischen Vereinfachung oder sogar zum Verzicht auf eine Äußerung7.

${ }^{7}$ In seiner Studie hat Zöfgen (2001: 99) auch festgestellt, dass das Kollokationslernen nicht durchs Lesen erfolgt, was er auf die „einseitige Rezeptionshaltung“ zurückführt, bei dem sich Fremdsprachenlernende auf das globale Leseverstehen konzentrieren. 
Diese Ergebnisse decken sich mit denen aus der Studie von Sadoughvanini und Shamsudin (2010), aus der hervorgeht, dass sich ein mangelhaftes bzw. mangelndes Kollokationswissen auf die Qualität der mündlichen Äußerung auswirkt. Wenn Fremdsprachenlernende manche Kollokationen, die sie in ihrer Äußerung gerne gebrauchen würden, nicht kennen, dann produzieren sie viel längere Sätze, weil sie die in ihrem mentalen Lexikon nicht verfügbaren Kollokationen zu umschreiben versuchen. Dadurch verliert ihre Aussage an Eindeutigkeit und Präzision. Auch die Wahrscheinlichkeit, lexikalische Fehler (Kollokationsfehler) zu machen, steigt.

Mangelhafte Kollokationskenntnisse führen also zu Kollokationsfehlern in mündlichen Äußerungen. Dies bestätigt die von Shamsudin, Sadoughvanini und Zaid (2013) durchgeführte Studie, in der vorbereitetes und unvorbereitetes Sprechen der Fremdsprachenlernenden analysiert wurde. Darin wurde festgehalten, dass im improvisierten Sprechen lexikalische Kollokationsfehler $67 \%$ aller Fehler ausmachten, wobei sich der Wert beim vorbereiteten Sprechen auf $65 \%$ belief. Im Falle der beiden Sprecharten betrug die Anzahl der L1-bedingten Kollokationsfehler über $80 \%$ der Fehler, weil die Probanden zielsprachliche Kollokationen nach dem Muster der L1 gebildet hatten. Aus der Studie geht hervor, dass der Gebrauch von fremdsprachlichen Kollokationen in der mündlichen Sprachproduktion den Fremdsprachenlernenden große Probleme bereitet und dass bei einem schwach entwickelten Kollokationswissen (vielleicht sogar schwach entwickelten Kollokationsbewusstsein) die Vorbereitung einer mündlichen Äußerung die Anzahl der nicht korrekt gebrauchten Kollokationen nicht reduziert.

Davon, dass zum korrekten Sprechen ein Kollokationswissen notwendig ist, zeugt die Studie von Attar und Allami (2013: 1076), in der die Forscher einen Zusammenhang zwischen dem Kollokationswissen und dem Gebrauch von Kollokationen festhalten konnten. Der positive Einfluss des Kollokationsunterrichts auf die Verwendung von Kollokationen wird damit erklärt, dass Fremdsprachenlernende beim Sprechen auf fertige, ihnen schon bekannte "Versatzstücke“ zurückgreifen, was zur schnelleren Sprachverarbeitung und somit zur besseren Sprachproduktion führt. Aus der Studie von Westerbrook (2015: 59f.) geht jedoch hervor, dass sich im Allgemeinen keine Korrelation zwischen der Kollokationskompetenz und der Sprachgewandtheit erfassen lässt. Interessant ist auch seine Beobachtung, dass Fremdsprachenlernende beim Gebrauch bestimmter Fachsprachen viele fachbereichsspezifische Kollokationen gebrauchen und dann auch flüssiger sprechen.

An dieser Stelle können noch die Überlegungen von Hill (1999) herangezogen werden, obwohl in seinem Beitrag kein Hinweis auf eine empirische Studie dazu zu finden ist. Er verweist auf einige Vorteile der Kollokationskenntnis auf die mündliche Äußerung. Laut ihm sind Kollokationen ein 
Schlüssel zum flüssigen Sprechen, denn der Abruf von längeren lexikalischen Chunks erlaubt dem Fremdprachenlernenden die Konzentration auf den Inhalt, wodurch der Stress bei der Suche nach Einzelwörtern, zu denen man in einem weiteren Schritt nach einem passenden lexikalischen Partner suchen muss, abgebaut wird. Des Weiteren beherrscht man beim Lernen von Kollokationen ein Betonungsschema für die ganze Phrase, was sich dann positiv auf die mündliche Äußerung auswirkt (Hill 1999: 5).

Aufgrund der oben dargestellten Untersuchungen kann man die Hypothese aufstellen, dass eine gut entwickelte Kollokationskompetenz die Qualität der mündlichen Äußerung positiv beeinflussen kann. Wenn Fremdsprachenlernende Kollokationen kennen, können sie von diesen Gebrauch machen. Sie müssen sich dann nicht darüber Gedanken machen, wie eine bestimmte Kollokation in der jeweiligen Fremdsprache lauten könnte. Das Erlernen von Kollokationen erlaubt einen Rückgriff auf feste Satzbausteine. Der Fremdsprachenlernende kann sich dann besser auf den Inhalt konzentrieren, weil sein Denken sich nicht zu stark auf die Zusammenstellung der Wörter in ein korrektes Syntagma richten muss.

\subsection{Kollokationskompetenz vs. Schreiben}

Die ersten empirischen Studien zur Wechselwirkung zwischen der Kollokationskompetenz und Schreibfertigkeit basierten auf dem Vergleich schriftlicher Texte, die von Muttersprachlern und Nicht-Muttersprachlern verfasst wurden (vgl. Granger 1998; Howarth 1998; Nesselhauf 2003). Aus diesen Studien geht hervor, dass Fremdsprachenlernende im Vergleich zu den Muttersprachlern viel seltener auf vorgefertigte Strukturen, d.h. Kollokationen und Idiome zurückgreifen und viel öfter Einzelwörter als Bausteine der Texte gebrauchen. Dies bedeutet, dass Fremdsprachenlernende auf das offene Prinzip der Sprache zurückgreifen, d.h. meistens nur freie Wortverbindungen gebrauchen, die sie selbstständig aus den Einzelwörtern bauen (können). Wenn sie überhaupt fremdsprachliche Kollokationen gebrauchen, dann handelt es sich zum einen um kongruente Kollokationen, die aus den gleichen Wörtern als Kollokationsglieder wie in ihrer Erstsprache zusammengestellt werden, zum anderen um hochfrequente Kollokationen, die sie in ihren Texten wiederholen (Durrant \& Schmitt 2009: 174f.).

Betonenswert ist jedoch die Tatsache, dass sich keine Korrelation zwischen der fremdsprachlichen Kompetenz und dem korrekten Gebrauch von Kollokationen in geschriebenen Texten festhalten lässt (Howarth 1998: 173f.), weil auch Fremdsprachenlernende mit sehr guten Fremdsprachenkenntnissen Kollokationsfehler machen. Diese resultieren aus Überschneidungen 
und Vermischungen. Dies bedeutet, dass Fremdsprachenlernende beim Schreiben zwei bedeutungsähnliche Kollokationen vermischen und ein Element aus einer Kollokation und das nächste aus einer anderen Kollokation nehmen und zu einer Quasi-Kollokation verbinden. Darüber hinaus liegt die wichtigste Ursache für Kollokationsfehler in der L1, wobei sich eine höhere Interferenz bei inkongruenten als bei kongruenten Kollokationen beobachten lässt (Nesselhauf 2003: 234ff.).

Nur eine Studie hat bestätigt, dass das Kollokationswissen mit dem Gebrauch von Kollokationen korreliert, d.h. je höher das Kollokationswissen der Fremdsprachenlernenden ist, desto öfter gebrauchen sie Kollokationen in geschriebenen Texten (Bazzaz \& Abd Samad 2011: 161). Der Autorin des Beitrags sind keine weiteren Studien dazu bekannt. Man kann jedoch (zum einen in Anlehnung an die gerade erwähnte empirische Studie, zum anderen stützen sich diese Annahmen auf Überlegungen der Autorin) vermuten, dass eine gut entwickelte Kollokationskompetenz in ihrer Dreigliedrigkeit den Fremdsprachenlernenden das Zurückgreifen auf das Idiomprinzip erlaubt bzw. ermöglicht. Kollokationskenntnis impliziert den häufigeren Gebrauch von Kollokationen und zwar der themenspezifischen. Wenn diese als zuvor gespeicherte Chunks in der richtigen Form abgerufen werden, steigt die Chance ihres korrekten Gebrauchs. Ihre Verwendung, d.h. der Verzicht auf die Umschreibung der Kollokationen, führt außerdem zur Präzision des sprachlichen Ausdrucks.

\section{ZUSAMMENFASSENDE BEMERKUNGEN}

Bevor Schlussfolgerungen aus den obigen Darlegungen gezogen werden, sollen im Folgenden weitere Ergebnisse einer empirischen Studie herangezogen werden, in der man den Zusammenhang zwischen den Kollokationskenntnissen und ihrem Einfluss auf andere Sprachfertigkeiten erforschte. Yazdandoost, AmalSaleh und Kafipour (2014) gingen der Frage nach, ob sich anhand der Kollokationskenntnisse der Fremdsprachenlernenden ihre Lese-, Hör-, Sprech- und Schreibfertigkeiten prognostizieren lassen. Die Autoren der empirischen Studie kamen zu dem Schluss, dass eine signifikante Korrelation zwischen Kollokationskenntnissen und allen vier Sprachfertigkeiten festzuhalten ist. Je höher die Kollokationskenntnisse der Fremdsprachenlernenden sind, desto besser schneiden sie bei allen Sprachfertigkeiten ab. Die stärkste Korrelation wurde zwischen den Kollokationskenntnissen und der Sprechfertigkeit und die schwächste zwischen den Kollokationskenntnissen und dem Hörverstehen beobachtet. Auf Grund dieser Studie formulieren ihre Autoren die These, dass sich anhand der Kollokationskenntnisse 
die Leistungen der Fremdsprachenlernenden in allen Sprachfertigkeiten voraussagen lassen.

Der vorliegende Beitrag verfolgte das Ziel, den Zusammenhang zwischen der Kollokationskompetenz und anderen Kompetenzen zu erfassen. Im Zentrum des Interesses standen auf der einen Seite die Kollokationskompetenz und ihr Einfluss auf das Hör- und Leseverstehen sowie auf das Sprechen und Schreiben. Zum anderen sollten die bis dahin weltweit durchgeführten empirischen Studien vieler Wissenschaftler unter dem Blickwinkel analysiert werden, ob sich die Kollokationskompetenz durch die Arbeit an den vier Sprachfertigkeiten entwickeln lässt. Die oben präsentierten Ergebnisse vieler empirischer Studien zeigen, dass die Kollokationskompetenz in alle Sprachfertigkeiten involviert ist. Eine (in ihrer Dreigliedrigkeit) gut entwickelte Kollokationskompetenz kann den Fremdsprachenlernenden ein korrekteres Schreiben und Sprechen ermöglichen. Sie kann sich auch als hilfreich bei den Aufgaben zum Hör- und Leseverstehen erweisen. Diese hier formulierte Hypothese könnte bzw. sollte den Ausgangspunkt für weitere empirische Studien darstellen. Dabei wird die Meinung vertreten, dass die Kollokationskompetenz im Fremdsprachenunterricht noch stärker gefördert werden muss, denn diese wichtige Kompetenz entwickelt sich leider im Fremdsprachenunterricht nicht von selbst. Wie aus den Ergebnissen der oben beschriebenen Studien hervorgeht, führt das bloße Lesen und Hören von Texten nicht automatisch zur Internalisierung der in diesen Texten vorkommenden Kollokationen. Zur Entwicklung einer Kollokationskompetenz reicht es nicht aus, Fremdsprachenlernende einem kollokationsreichen Input auszusetzen. Kollokationen sind, worauf schon viele Wissenschaftler hingewiesen haben, in der Sprachrezeption unauffällig. Deshalb bedürfen sie der gezielten Aufmerksamkeit seitens der Fremdsprachenlernenden. Diese kann mittels verschiedener didaktischer Schritte bzw. Vorgehensweisen auf Kollokationen ausgerichtet werden.

Kollokationen müssen im Fremdsprachenunterricht stärker berücksichtigt werden. Wie dies zu bewerkstelligen ist, darüber machten sich schon viele Forscher seit Langem Gedanken. Ihre Darstellung geht jedoch über das Thema des vorliegenden Beitrags hinaus. An dieser Stelle werden nur als Beispiel zwei entgegengesetzte Meinungen herangezogen. Holderbaum (2003: 87) vertritt den Standpunkt, dass sich die fremdsprachliche Kollokationskompetenz „durch gezielte Übungen, die möglichst alle Wahrnehmungskanäle ansprechen, und durch einen intensiven Kontakt mit der Fremdsprache [...] erkennbar erweitern lässt." Demgegenüber ist Lütge (2000: 345) der Ansicht, dass sich die Kollokationskompetenz durch language awareness entwickeln lässt. Die Förderung des Kollokationsbewusstseins solle dazu führen, dass sich die Fremdsprachenlernenden beim Wortschatzlernen nicht 
nur auf Einzelwörter, sondern auf Mehrwortlexeme bzw. Wortverbindungen konzentrieren. Es bedarf jedoch weiterer Erforschung, welche didaktischen Schritte effizienter sind und besser bzw. schneller zur Entwicklung der Kollokationskompetenz beitragen.

\section{LITERATURVERZEICHNIS}

Attar, E. M. / Hamid A. (2013). The effects of teaching lexical collocations on speaking ability of Iranian EFL learners. Theory and practice in language studies, 3 (6), 1070-1079.

Bahns, J. (1997). Kollokationen und Wortschatzarbeit im Englischunterricht. Tübingen: Narr.

Bahns, J. / Sibilis, U. (1992). Kollokationslernen durch Lektüre. Neusprachliche Mitteilungen aus Wissenschaft und Praxis, 45 (3), 158-163.

Bazzaz, F. E. / Abd Samad, A. (2011). The use of verb noun collocations in writing stories among Iranian EFL learners. English Language Teaching , 4 (3), 158-163.

Dentisak, D. (2015). The effects of instruction of noticing collocation on Thai EFL learners. Journal of Advances in English Language Teaching, 3 (1), 1-11.

Durrant, P. / Schmitt, N. (2009). To what extent do native and non-native writers make use of collocations? International Review of Applied Linguistics in Language Teaching (IRAL) 47, 157-177.

Gabryś-Biskup, D. (1990). Some remarks on combinability: Lexical collocation. In: J. Arabski (Hrsg.), Foreign language acquisition papers (S. 31-44). Katowice: Uniwersytet Śląski.

Goudarzi, Z. / Moini, M. R. (2012). The effect of input enhancement of collocations in reading on collocation learning and retention of EFL learners. International Education Studies. 5 (3), 247-258.

Granger, S. (1998). Prefabricated patterns in advancer EFL writing: collocations and lexical phrases. In: A. P. Cowie (Hrsg.), Phraseology: theory, analysis and applications (S. 145-160). Oxford: Clarendon Press.

Hausmann, F. J. (1984). Wortschatzlernen ist Kollokationslernen. Zum Lehren und Lernen französischer Wortverbindungen. Praxis des Neusprachlichen Unterrichts, 3 (4), 395-406.

Hausmann, F. J. (2007). Die Kollokationen im Rahmen der Phraseologie - systematische und historische Darstellung. Zeitschrift für Anglistik und Amerikanistik, 55 (3), 217-234.

Henriksen, B. (2013). „Research on L2 learners' collocational competence and development a progress report". In: C. Bardel / C. Lindquist / B. Laufer (Hrsg.), L2 vocabulary acquisition, knowledge and use. New perspective on assessment and corpus analysis (S. 29-56). Eurosla. http://www.eurosla.org/monographs/EM02/Henriksen.pdf (Zugriff am: 15.08.2018).

Hill, J. (1999). Collocational competence. English Teaching professional 3-7. http:/ / www.google. $\mathrm{pl} / \mathrm{url}$ ?sa $=\mathrm{t} \& \mathrm{rct}=\mathrm{j} \& \mathrm{q}=\& \mathrm{esrc}=\mathrm{s} \& \mathrm{source}=$ web\&cd $=1 \& \mathrm{ved}=0 \mathrm{CDIQFjAA \& url}=\mathrm{http} \% 3 \mathrm{~A} \% 2 \mathrm{~F} \%$ 2Fwww.freewebs.com\%2Fenglish1-0\%2Fcompetence.doc\&ei=zefbUanEMMPIPLHvgLAO \&usg=AFQjCNFqqbxgguiNSE9OTYK-ea09UePgrw\&sig2=6MXGSKw_4gFWJ7seZ7JeeA\& bvm=bv.48705608,d.ZWU

Holderbaum, A. (2003). Kollokationen als Problemgrößen der Sprachmittlung. Trier: Wissenschaftlicher Verlag Trier.

Howarth, P. (1998). The phraseology of learners' academic writing. In: A. P. Cowie (Hrsg.), Phraseology: theory, analysis, and application (S. 161-186). Oxford: Clarendon Press.

Hsu, J. T. (2010). The effects of collocation instruction on the reading comprehension and vocabulary learning of Taiwanese College English Majors. The Asian EFL Journal Quarterly, $12(1), 47-87$. 
Hug, A. / Leupold, E. (2008). Kompetenzorientierung und Französischunterricht. In: B. Tesch / E. Leupold / O. Köller (Hrsg.), Bildungsstandards Französisch: konkret. Sekundarstufe I: Grundlagen, Aufgabenbeispiele und Unterrichtsanregungen. Berlin: Cornelsen.

Hulbert, J. (2015). Ist Kollokationsbewusstheit implizit zu vermitteln? - Eine Untersuchung zum Potenzial der Dictogloss-Aufgabe. In: B. Handwerker / R. Bäuerle / I. Doval / B. Lübke (Hrsg.), Zwischenräume: Lexikon und Grammatik im Deutschen als Fremdsprache (S. 171-189). Hohengehren: Schneider.

Khonamri, F. / Roostaee S. (2014). Does extensive reading combined with form-focused or meaning-focused activities affect lexical collocational knowledge of Iranian learners? Theory and Practice in Language Studies, 4 (5), 1038-1044.

Konecny, Ch. (2010). Kollokationen. Versuch einer semantisch-begrifflichen Annäherung und Klassifizierung anhand italienischer Beispiele. München: Martin Meidenbauer Verlagsbuchhandlung.

Krumm, H.-J. / Fandrych, Ch. / Hufeisen, B. / Riemer, C. (Hrsg.) (2010). Deutsch als Fremdund Zweitsprache. Ein internationales Handbuch. 1. Halbband. Berlin: Walter de Gruyter.

Lütge, Ch. (2000). Sprachbewusstheit und Wortschatzerwerb - zu Kollokationskompetenz fortgeschrittener Lerner des Englischen. In: K. Aguado / A. Hu (Hrsg.), Mehrsprachigkeit und Mehrkulturalität: Dokumentation des 18. Kongresses für Fremdsprachendidaktik, veranstaltet von der Deutschen Gesellschaft für Fremdsprachenforschung (DGFF) Dortmund, 4.-6. Oktober 1999 (S. 343-349). Berlin: PZV Pädagogischer Zeitschriftenverlag.

Lütge, Ch. (2002). Syntagmen und Fremdsprachenerwerb. (Ein Lernersprachenproblem). Frankfurt a.M.: Peter Lang.

Marton, W. (1977). Foreign vocabulary learning as a problem No. 1 of language teaching at the advanced level. Interlanguage Studies Bulletin, 2, 33-57.

Müller, T. (2011). Aware of collocations. Ein Unterrichtskonzept zum Erwerb von Kollokationskompetenz für fortgeschrittene Lerner des Englischen. Frankfurt am Main: Peter Lang.

Nam, H.-J. (2013). Pedagogical implications of teaching English collocations to Korean EFL Learners. Studies in English Education, 18 (1), 59-86.

Nesselhauf, N. (2003). The use of collocations by advanced learners of English and some implication for teaching. Applied Linguistics, 24 (2), 223-242.

Reder, A. (2001). Ein heißer Tipp - oder Kollokationslernen durch eine Ganzschrift. In: A. Häcki Buhofer, / P. Durčo (Hrsg.), Wortschatz: Aneignung und Unterricht. IDT Publikation 2001. https:/ / sites.google.com/site/kollokator/home/aufsaetze (Zugriff am: 12.08.2018).

Reder, A. (2002). Eine Aufgaben- und Übungstypologie zur Entwicklung der Kollokationskompetenz von DaF-Lernern. Jahrbuch der ungarischen Germanistik, 293-311.

Reder, A. (2006). Kollokationen in der Wortschatzarbeit. Wien: Praesens.

Rössler, A. (2010). Kollokationskompetenz fördern im Fremdsprachenunterricht - Ein Plädoyer. Die Neueren Sprachen, 1, 54-66.

Sadoughvanini, S. / Shamsudin S. B. (2010). Impromptu speech: EFL learners' problems in collocation and L1 interference. EDUPRESS: Education Postgraduate Research Seminar 2010, UTM-Faculty of Education, Johor, Malaysia, 322-335. http://eprints.utm.my/id/eprint/ 14938/1/Impromptu_Speech.pdf (Zugriff am: 20.08.2018).

Shamsudin, S. / Sadoughvanini, S. / Zaid Y. H. (2013). Iranian' EFL learners collocational errors in speaking skill. Procedia - social and behavioral sciences 70, 1295-1302.

Siepmann, D. (2002). Eigenschaften und Formen lexikalischer Kollokationen. Wider ein zu enges Verständnis. Zeitschrift für französische Sprache und Literatur, 112 (3), 240-263.

Siepmann, D. (2004). Kollokationen und Fremdsprachenlernen. Imitation und Kreation, Figur und Hintergrund. Praxis Fremdsprachenunterricht, 2, 107-113. 
Targońska, J. (2014). Der Kollokationsbegriff im Lichte der geschichtlichen Entwicklung. Kwartalnik Neofilologiczny, 4, 695-717.

Targońska, J. / Stork, A. (2013). Vorschläge für ein neues Modell zur Beschreibung und Analyse lexikalischer Kompetenz. Zeitschrift für Fremdsprachenforschung, 24 (1), 71-108.

Targońska, J. / Stork, A. (2017). Wie lässt sich Kollokationskompetenz evaluieren? Untersuchungsmethoden zur Erfassung von Kollokationskompetenz und deren problematische Bereiche. Linguistische Berichte 250, 219-245.

Tschirner, E. (2010). Wortschatz. In: H.-J. Krumm et al. (Hrsg.), Deutsch als Fremd- und Zweitsprache. Ein internationales Handbuch. 1. Halbband (S. 236-245). Berlin: Walter de Gruyter.

Viehweger, D. (1987). Kollokationen. In: W. Neumann / B. Techtmeier (Hrsg.), Bedeutungen und Ideen in Sprachen und Texten (S. 227-237). Berlin: Akademie-Verlag.

Westerbrook, P. N. (2015). Talk about mouth speculums: collocational competence and spoken fluency in non-native English-speaking university lectures (Unveröffentlichte Doktorarbeit). University of Birmingham, Edgbaston / Birmingham. https://www.academia.edu/25229275/TALK_ ABOUT_MOUTH_SPECULUMS_COLLOCATIONAL_COMPETENCE_AND_SPOKEN_ FLUENCY_IN_NON-NATIVE_ENGLISH-SPEAKING_UNIVERSITY_LECTURERS.

Yazdandoost Z. / AmalSaleh, E. / Kafipour, R. (2014). The relationship among collocational knowledge and listening, speaking, reading and writing proficiency of Iranian EFL learners. Language, Individual \& Society, 8, 408-419.

Zöfgen, E. (2001). Lexikalische Zweierverbindungen: „Vertraute Unbekannte“ im mentalen Lexikon germanophoner Französischlerner. Französisch heute, 1, 89-107.

Received: 30.08.2018; revised: 9.02.2019 